\title{
Mutation screening of TSC1 and TSC2 genes in Chinese Han children with tuberous sclerosis complex
}

\author{
C.R. Mi ${ }^{1}$, H. Wang ${ }^{2}$, H. Jiang ${ }^{3}$, R.P. Sun ${ }^{1}$ and G.X. Wang ${ }^{2}$ \\ 'Department of Paediatrics, Qilu Hospital of Shandong University, \\ Jinan, China \\ ${ }^{2}$ Medical Institute of Paediatrics, \\ Qilu Children's Hospital of Shandong University, Jinan, China \\ ${ }^{3}$ Key Laboratory of Cardiovascular Remodeling and Function Research, \\ Qilu Hospital of Shandong University, Jinan, China \\ Corresponding author: G.X. Wang \\ E-mail: wanggxemail@qq.com
}

Genet. Mol. Res. 13 (1): 2102-2106 (2014)

Received January 8, 2014

Accepted March 12, 2014

Published March 24, 2014

DOI http://dx.doi.org/10.4238/2014.March.24.14

\begin{abstract}
Tuberous sclerosis complex (TSC) is an autosomal dominant neurogenetic disorder caused by mutations in the TSCl or TSC2 genes and is frequently associated with hamartoma formation in multiple organ systems. Here, we report two novel mutations in the TSC2 gene, including a splicing mutation (IVS $29+1 \mathrm{G}>\mathrm{C}$ ) in intron 29 and a deletion/insertion mutation (C.5090-5092delCCA- inAG) in exon 39 in two Chinese Han children with TSC whose first clinical manifestation was seizure. The identification of these two mutations confirmed the diagnosis of TSC and expands the spectrum of TSC2 mutations causing TSC.
\end{abstract}

Key words: TSC1 gene; TSC2 gene; Tuberous sclerosis complex; Mutation 


\section{INTRODUCTION}

Tuberous sclerosis complex (TSC; MIM \#191100 and MIM \#613254) is an autosomal dominant, multisystem, neurocutaneous disorder characterized by the formation of hamartomas in multiple organs such as the brain, kidneys, skin, heart, and lungs (Aronow et al., 2012; Amin and O'Callaghan, 2013). The neurological features of TSC are associated with the highest morbidity including intractable epilepsy, autism, cognitive disability, hydrocephalus, focal neurological deficits, and neurobehavioral abnormalities (Crino, 2013). The pathogenic genes include TSC1 and TSC2, which are tumor-suppressor genes that map to chromosomes 9q34 and 16p13.3, respectively. Hamartin and tuberin, which are encoded by TSC1 and TSC2, form a complex and play an important role in cell proliferation and migration. The incidence of TSC is estimated to be $8-9$ per 100,000 individuals, and approximately $65 \%$ of cases can be identified based on sporadic mutations (Lendvay and Marshall, 2003), including a broad range of small insertions and deletions, single-base substitutions resulting in nonsense codons, missense changes, or splicing mutations, and larger genomic deletions. Here, we report two novel mutations in TSC2, including a splicing mutation (IVS $29+1 \mathrm{G}>\mathrm{C}$ ) in intron 29 and a deletion/insertion mutation (C.5090-5092delCCA-inAG) in exon 39, which were identified by screening the TSC1 and TSC2 genes in two Chinese Han children with TSC.

\section{MATERIAL AND METHODS}

\section{Patients}

Patients with sporadic TSC included one female and one male from the Qilu Children's Hospital of Shandong University. The children were from different families and fulfilled the definitive TSC criteria of the Tuberous Sclerosis Consensus Conference (Roach et al., 1999). Both subjects were of the Chinese Han ethnic group; a detailed clinical evaluation including medical history and physical examination were obtained for comparison of standardized clinical data. This study was approved by the research ethics committee of Qilu Hospital of Shandong University, and consent was obtained from the parents of the subjects. The two cases were both sporadic, and none of their relatives had TSC.

Case 1 was an 8-month-old female. She suffered from nodding and frequent blinking when she was 1-month-old and received medical attention for frequent seizures in July 2012. Upon physical examination, her height was $69 \mathrm{~cm}$, weight was $8 \mathrm{~kg}$, and hypomelanotic macules were present on her head (Figure 1a), face, back, and legs. An electroencephalogram showed characteristic hypsarrhythmia, slow sharp-waves, slow spike-waves, and multispike slow waves. Cranial computed tomography showed subependymal nodules along the lateral walls of the lateral ventricles and cerebral cortex.

Case 2 was a 9-month-old male. He experienced seizures 4 times per day at the age of 4 months. Medical attention was sought one month later and the seizures were controlled by oxcarbazepine. However, a moderate developmental delay was demonstrated. Abdominal white macules were found in the face and trunk. Subependymal nodules were identified on a brain magnetic resonance imaging scan (Figure 1b). The electroencephalogram revealed sharp waves and sharp-slow waves in the cortical region. His parents and other family members were not affected by TSC. 

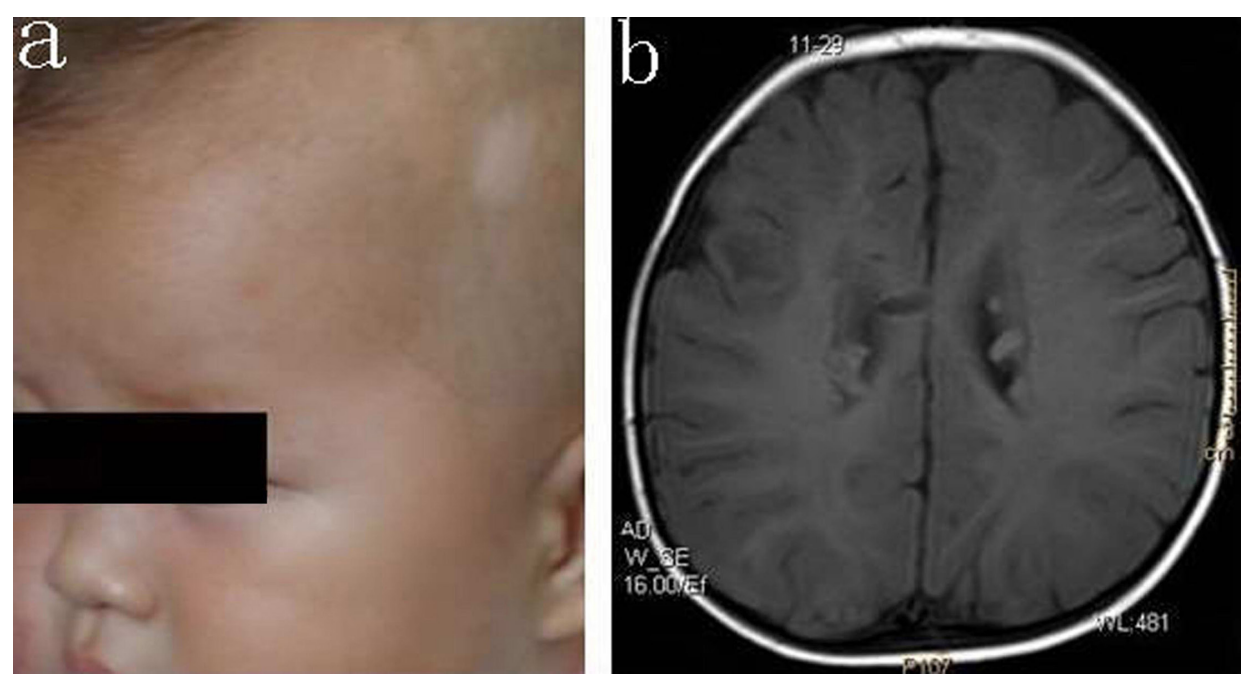

Figure 1. Major features of the patients with TSC. A. Hypomelanotic macules on the head of case 1. B. Cranial CT scan of case 2: subependymal calcifications along the lateral walls of the lateral ventricles.

\section{Methods}

First, $3 \mathrm{~mL}$ peripheral blood was collected from the patients, their parents, and 100 unrelated normal controls without any physical/psychological diseases. In addition, no history of TSC was found in first-degree relatives of the controls. Genomic DNA was extracted using the TIANamp Blood DNA Kit (Tiangen Biotech Co.; Beijing, China). Polymerase chain reaction (PCR) primers and running conditions for each exon are available in a previous report (Wang et al., 2013). Twenty-five pairs of PCR primers for exons 1-23 of the TSC1 gene and 36 pairs of PCR primers for exons $0-41$ of the TSC2 gene were prepared to obtain the amplicons. The entire coding region together with the exon-intron boundaries was amplified by PCR. The PCR was run on each exon in a $50 \mu \mathrm{L}$ total sample volume containing 300 ng genomic DNA, $2.0 \mu \mathrm{L}$ of each primer $(10 \mu \mathrm{M})$, and $25 \mu \mathrm{L} 2 \mathrm{X}$ PCR Mastermix (Tiangen Biotech Co.) in a GeneAmp 9700 thermal cycler (Perkin-Elmer; Waltham, MA, USA). The PCR amplification began with a denaturing step at $94^{\circ} \mathrm{C}$ for 3 min, followed by 31 cycles of denaturing at $94^{\circ} \mathrm{C}$ for $35 \mathrm{~s}$, annealing at 50 to $62^{\circ} \mathrm{C}$ for $35 \mathrm{~s}$, extension at $72^{\circ} \mathrm{C}$ for $55 \mathrm{~s}$, and final extension at $72^{\circ} \mathrm{C}$ for $5 \mathrm{~min}$. Next, gel purification of the products was performed using an agarose gel DNA purification kit (Tiangen Biotech Co.). Forward and reverse primers were used to sequence the purified PCR products, and automated sequencing was performed on an ABI 377 sequencer. Each mutation was confirmed by sequencing individual amplicons.

Once a mutation within an exon/intron was detected in the patients, this exon/intron was PCR-amplified specifically from the DNA of their parents and 100 unrelated normal controls.

\section{RESULTS}

In the current study, we performed mutational analysis of the entire coding regions as well as the exon-intron boundaries of TSC1 and TSC2 genes in individuals with TSC and 
their family members using PCR and DNA sequencing. We checked the single-nucleotide polymorphism lists at the NCBI Entrez SNP database and compared the findings to those of 100 healthy controls when identifying the mutation. No mutations were found in the TSC1 gene, but 2 novel mutations were confirmed in the TSC2 gene. A splicing mutation in case 1 was identified as a nucleotide transition of $\mathrm{G}$ to $\mathrm{C}$ at position c.3610+1 in TSC2 intron 29 (IVS $29+1 \mathrm{G}>\mathrm{C}$ ) in this patient (Figure 2a). A deletion/insertion mutation (C.5090-5092delCCAinAG) in exon 39 (Figure $2 \mathrm{~b}$ ) was detected in case 2 and resulted in a frameshift mutation.
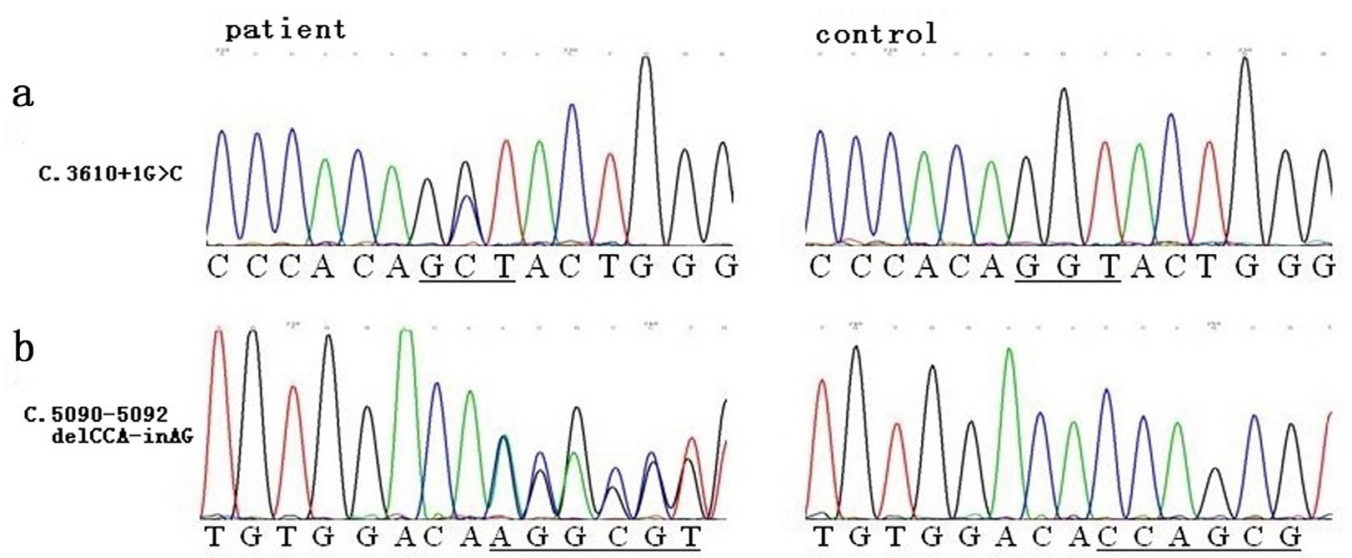

Figure 2. Sequence chromatograms of TSC2 gene in patients and controls. The sites of mutations (left column) in case 1(a), case 2 (b) are shown compared with the corresponding sites in controls (right column).

Both mutations were novel and the screening showed that neither of these mutations was present in the patients' parents or the 100 unrelated normal controls. Thus, these 2 mutations may be pathogenic and were regarded as TSC-causing mutations.

\section{DISCUSSION}

TSC is a genetic disease caused by a wide spectrum of mutations in the TSC1 or TSC2 genes, in which formation of hamartomas affects multiple organ systems and results in variable clinical expressions. Infantile spasms, intractable epilepsy, cognitive disabilities, and autism are the main clinical features in the central nervous system of patients suffering from TSC (Wataya-Kaneda et al., 2013; Michelozzi et al., 2013; Hsieh et al., 2013).

The genetic basis of TSC is heterogeneous in 2 unlinked causative genes, TSC1 and TSC2. Human TSC1 consists of 23 exons and encodes a 130-kDa protein: TSC1 (or hamartin). TSC2 consists of 41 exons spanning $45 \mathrm{~kb}$ of genomic DNA and encodes a 5.5-kb mRNA. The encoded protein, tuberin (or TSC2), contains 1807 amino acids (198 kDa), and has the following 7 functional domains from the $\mathrm{N}$-terminus to the $\mathrm{C}$-terminus: leucine zipper domain encoded by exon 3; coiled coil domain 1 encoded by exon 10; CCD2 encoded by exon 26; transcription activation domain 1 encoded by exons 29-30; GAP domain encoded by exons 34-38; TAD2 encoded by exons 39-40; and a calmodulin-binding domain encoded by exons 40-41 (Krymskaya, 2003; Napolioni and Curatolo, 2008). Over 900 mutations in TSC1 and TSC2 have been reported (Padma and Dalal, 2012; Jang et al., 2012). 
In our study, 2 mutations were identified in the TSC 2 gene. One mutation was a splicing mutation in TSC2 intron 29 (C. $3610+1 \mathrm{G}>$ C) in case 1. In silico analysis indicated that this mutation abolishes the donor splice site, leading to cryptic site activation downstream of the normal site in intron 29 , resulting in a translation of intronic sequence 29 along with frameshift changes. The functional domain transcription activation domain 1, encoded by exons 29-30, is predictably influenced by this abnormal splicing, impairing transcription activity. The 2 nd mutation was a deletion/insertion mutation (C.5090-5092delCCA-inAG) in exon 39, which resulted in a substitution of lysine $(\mathrm{K})$ for threonine $(\mathrm{T})$ at codon 1697 following a frameshift influencing exons 39,40 , and 41 . Accordingly, the peptide chain was clearly altered, and the function of transcription activation domain 2 and calmodulin-binding domain was predictably destroyed. We did not observe these 2 mutations in the single-nucleotide polymorphism database or the human gene mutation database. In these 2 cases, dysfunction of 1 or more functional domains induced the TSC phenotype.

In summary, the PCR and DNA sequence analyses revealed 2 mutations in the TSC2 gene in 2 sporadic TSC cases in Chinese Han children. Both mutations are novel. The identification of these 2 mutations confirmed the diagnosis of 2 children with TSC and expanded the spectrum of TSC2 mutations causing TSC.

\section{ACKNOWLEDGMENTS}

The authors are deeply grateful to the patients with TSC for their participation and cooperation in this study, as well as to Da-Wei Wang for rewriting the manuscript.

\section{REFERENCES}

Amin S and O'Callaghan FJ (2013). Glossal hamartoma in tuberous sclerosis. Arch. Dis. Child. 98: 161.

Aronow ME, Nakagawa JA, Gupta A, Traboulsi EI, et al. (2012). Tuberous sclerosis complex: genotype/phenotype correlation of retinal findings. Ophthalmology 119: 1917-1923.

Crino PB (2013). Evolving neurobiology of tuberous sclerosis complex. Acta Neuropathol. 125: 317-332.

Hsieh DT, Jennesson MM and Thiele EA (2013). Epileptic spasms in tuberous sclerosis complex. Epilepsy Res. 106: 200-210.

Jang MA, Hong SB, Lee JH, Lee MH, et al. (2012). Identification of TSC1 and TSC2 mutations in Korean patients with tuberous sclerosis complex. Pediatr. Neurol. 46: 222-224.

Krymskaya VP (2003). Tumour suppressors hamartin and tuberin: intracellular signalling. Cell Signal. 15: 729-739.

Lendvay TS and Marshall FF (2003). The tuberous sclerosis complex and its highly variable manifestations. J. Urol. 169: $1635-1642$.

Michelozzi C, Di LG, Galli F, Silva BF, et al. (2013). Subependymal nodules and giant cell tumours in tuberous sclerosis complex patients: prevalence on MRI in relation to gene mutation. Childs Nerv. Syst. 29: 249-254.

Napolioni V and Curatolo P (2008). Genetics and molecular biology of tuberous sclerosis complex. Curr. Genomics 9: 475-487.

Padma PT and Dalal AB (2012). Tuberous sclerosis: diagnosis and prenatal diagnosis by MLPA. Indian J. Pediatr. 79: 1366-1369.

Roach ES, DiMario FJ, Kandt RS and Northrup H (1999). Tuberous Sclerosis Consensus Conference: recommendations for diagnostic evaluation. National Tuberous Sclerosis Association. J. Child. Neurol. 14: 401-407.

Wang GX, Wang DW, Yi CY, Qu JS, et al. (2013). Mutational analyses of the TSC1 and TSC2 genes in cases of tuberous sclerosis complex in Chinese Han children. Genet. Mol. Res. 12: 1168-1175.

Wataya-Kaneda M, Tanaka M, Hamasaki T and Katayama I (2013). Trends in the prevalence of tuberous sclerosis complex manifestations: an epidemiological study of 166 Japanese patients. PLoS One 8: e63910. 